\section{Wie lange sind Ohrentropfen haltbar?}

\author{
Antibiotikahaltige Präparate werden bei bakterieller Otitis häufig zur \\ topischen Behandlung eingesetzt - meist nur über wenige Tage. Wie lange \\ diese Medikamente tatsächlich haltbar sind, überprüfte ein kanadisches \\ Expertenteam.
}

E s liegen kaum genaue Daten darüber vor, wie lange antibiotikahaltige Externa zur Behandlung einer Otitis externa oder media wirksam sind. Ebenso interessant ist die Frage, ob von Patienten benutzte Präparate kontaminiert sind und möglichst schnell entsorgt werden müssen.

Kanadische Forscher testeten zwei sehr häufig eingesetzte Ohrentropfenpräparate, eines mit Ciprofloxacin und Dexamethason, ein anderes mit Gentamycin und Betamethason. Diese zwei topischen Präparate werden vor allem gegen die Haupterreger Pseudomonas aeruginosa und Staphylococcus aureus eingesetzt. Die Wirksamkeit der Präparate wurde getestet, indem sie auf Agarplatten aufgetragen wurden, die mit diesen beiden Keimen inokuliert waren. In einem weiteren Ansatz wurden längere Zeit geöffnete Präparate auf Kontamination untersucht. Schließlich testeten die Forscher den Inhalt von Medikamentenfläschchen, die Patienten mit einer Otitis (Ausfluss aus dem Ohr, alle Kulturen mit positivem Bakteriennachweis) eine Woche lang benutzt hatten.

Die Wirksamkeit der Präparate blieb über einen Beobachtungszeitraum von vier Monaten erhalten. Im gesamten Zeitverlauf war keine signifikante Reduktion der Inhibitionszone auf den Agarplatten erkennbar. Dies galt für beide Präparate - sowohl gegen Pseudomonas aeruginosa als auch gegen Staphylococcus aureus. Einmal geöffnete Medikamentenfläschchen blieben bis zu vier Mona- ten ohne Keimbefall. Die auf Agarplatten inkubierten Lösungen zeigten keinerlei Wachstum von Mikroorganismen. Das Gleiche galt für sämtliche Präparate, die von Patienten mit einer bakteriellen Otitis über eine Woche lang ordnungsgemäß benutzt worden waren.

Fazit: In dieser Studie blieben Ciprofloxacin- und Gentamycin-haltige Ohrentropfen gegen Pseudomonas aeruginosa und Staphylococcus aureus über vier Monate wirksam. Eine Kontamination länger geöffneter oder sogar von Patienten mit bakterieller Otitis benutzter Medikamente konnte nicht nachgewiesen werden. af

Clark MPA et al. The shelf life of antimicrobial ear drops. Laryngoscope 2010;3: 565-9

\section{Kommentar}

In der Einleitung der obigen Veröffentlichung wird die Anwendung von antibiotikahaltigen Ohrentropfen als Standardtherapie für ein sezernierendes Ohr aufgeführt, was sowohl für die Otitis externa als auch für die Otitis media gelte. Diese Formulierung sollte nicht unkommentiert übernommen werden, da die bekannten Risiken einer inadäquaten Therapie (v.a. Langzeittherapie) abgeschwächt eingestuft werden. Auch wenn, wie angegeben, Pseudomonas aeruginosa und Staphylococcus aureus mit die häufigsten Erreger der entzündlichen Ohrerkrankungen mit Otorrhö sind, gilt bei der Entscheidung zur topischen Anwendung von Antibiotika eine diagnostische Abwägung anderer Ur- sachen. Letztlich ziehen diese alternative Behandlungsverfahren nach sich. Es wäre gut, wenn im Zusammenhang mit der Veröffentlichung der Studienergebnisse darauf noch einmal verwiesen worden wäre.

Das Ergebnis der Studie räumt mit der Unsicherheit über die Haltbarkeit und damit auch mit der Unsicherheit der noch möglichen Anwendung geöffneter OriginalAbpackungen antibiotikahaltiger Ohrentropfen auf. Es wird klar aufgezeigt, dass auch noch nach vier Monaten die bakterizide Wirkung der Lösungen vorhanden ist. Daraus leiten sich folgende Überlegungen ab: Die Empfehlungen der Hersteller, die meist nur eine sechswöchige oder kürzere Haltbarkeit empfehlen, sollte revidiert werden, und der Inhalt der in Praxen und klinischen Behandlungseinrichtungen geöffneten Originalverpackungen kann somit zukünftig länger verwendet werden, was Kosten sparen hilft.

Die vorliegende Information der Studie darf jedoch dem Patienten nicht unkommentiert zugeleitet werden, da dieser möglicherweise die Gefahrlosigkeit einer Langzeitanwendung ableitet. Wiederholte Selbstbehandlungen mit nicht verbrauchten Ohrentropfenabpackungen könnten die Folge sein. Die Gefahr einer unkontrollierten ototoxischen Wirkung, die gerade bei Gentamicin-haltigen Tropfen und bestehender Otitis media kritisch ist, wird verkannt. Auch steigen allergisierende Wirkungen und Resistenzentwicklungen proportional zur Anwendungsdauer, was bei unkontrollierter langdauernder oder häufig wiederholter Selbstbehandlung ein hohes Risiko darstellt.

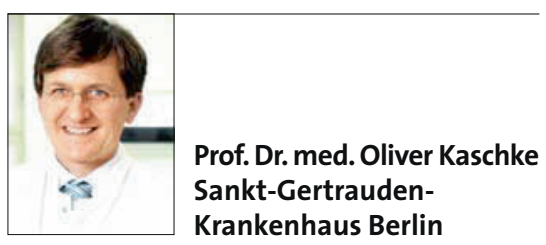

